\title{
Stable periodic motion of a controlled segmented leg model of pedal locomotion with inelastic ground-foot collision
}

\author{
Ambrus Zelei $\odot$. Bernd Krauskopf • \\ Petri T Piiroinen · Tamás Insperger
}

Received: 7 June 2018 / Accepted: 20 March 2019 / Published online: 10 April 2019

(C) The Author(s) 2019

\begin{abstract}
Human and robotic legged locomotion can be described with complex multi-degree-of-freedom dynamic models, whose bifurcation or parameter analysis may explain some features of typical patterns during motion. In this paper, we focus on the effect of kinematic parameters and foot placement techniques on the ground-foot impact intensity. The work is based on a multibody dynamic model of a segmented leg, which possesses some fundamental characteristics of locomotion systems: (a) distinct topology in the flight and ground phases; (b) kinetic energy absorption due to partially/fully inelastic ground-foot collision; (c) an active
\end{abstract}

A. Zelei $(\square)$

MTA-BME Research Group on Dynamics of Machines and Vehicles, Múegyetem rkp. 3-5., Budapest, Hungary e-mail: zelei@mm.bme.hu

\section{A. Zelei · T. Insperger}

MTA-BME Lendület Human Balancing Research Group, Múegyetem rkp. 3-5., Budapest, Hungary

B. Krauskopf

Department of Mathematics, University of Auckland, Private Bag 92019, Auckland 1142, New Zealand

e-mail: b.krauskopf@auckland.ac.nz

P. T. Piiroinen

School of Mathematics, Statistics and Applied

Mathematics, National University of Ireland Galway,

University Road, Galway H91 TK33, Ireland

e-mail: petri.piiroinen@nuigalway.ie

T. Insperger

Department of Applied Mechanics, Budapest University of

Technology and Economics, Múegyetem rkp. 3-5.,

Budapest, Hungary

e-mail: insperger@mm.bme.hu control strategy for maintaining a prescribed mechanical energy level; and (d) different control strategies for the flight and ground phases. We obtain a quantitative measure for the foot collision intensity by analytic calculations. The pre-impact velocity conditions are obtained from a hopping three-segmented planar leg model that imitates pedal locomotion. The singlelegged model contains the foot, the shank, the thigh and a reaction wheel attached to the hip, which models the effect of the upper body. The existence of stable periodic motion associated with hopping is shown for a wide range of parameters by means of finding suitable control torques in the ankle, the knee and the hip joint. The parameters of the linear feedback controller are tuned to optimize different cost functions, such as running speed, energy efficiency and impact intensity. We also investigate how the stability of the periodic motion depends on the control gains.

Keywords Legged locomotion · Human balancing · Constrained motion space kinetic energy (CMSKE) . Piecewise-smooth dynamical systems · Periodic orbits

\section{Introduction}

Analyzing the dynamics of legged locomotion is a challenging task for several reasons. The associated mechanical models involve multiple degree-offreedom (DoF) systems, varying topology, alternating over- and under-actuated [1] motions related to 
the different phases of walking or running, nonlinearities related to geometrical configurations [2], and non-smoothness at foot impacts [3-9]. Moreover, such models often involve some kind of motor pattern generator [10]. Underlying feedback mechanisms introduce additional components into models, such as reaction time delay, sensory uncertainties and the saturation of the forces exerted by the muscles [11]. Furthermore, the control concept employed by the nervous system to keep the body segments on the desired path is not exactly known.

Still, there are high DoF models in the literature for legged locomotion, which are used for the interpretation of experimental results, such as the calculation of joint forces derived from motion capturing $[12,13]$. These models take the geometry of the human body into account precisely; however, important aspects such as balance, motion pattern generation and control are generally not included.

Another group of models includes the balancing and control strategy of the brain; however, their geometry is reasonably simplified. Such models help one understand how the nervous system keeps the body in balance, how the energy level is maintained and how the motion patterns are generated during locomotion. The balancing process can be understood better by including a controller that mimics the operation of the nervous system [14]. For instance, the spring-loaded inverted pendulum (SLIP) model in [15] involves a sophisticated control algorithm that takes stability and control issues into consideration, while the other elements of the model are excessively simplified compared to the human body. The model in [16], on the other hand, features a geometry very similar to the human leg, but the model still neglects the mass of leg segments. Consequently, the important issues of ground-foot collision and impact-induced energy absorption are missed. In fact, a considerable amount of energy-demand in locomotion is related to ground-foot impact. Furthermore, foot impact is the main source of injuries when walking and running.

In this paper, we present the stability analysis of the three-segmented controlled leg model developed in [17]. The model describes a stable periodic motion associated with hopping. The goal is to present a dynamic analysis of a moderately complex model, for which the number of parameters is still small enough to be treated with qualitative mathematical methods. The new contribution of the present paper is that the local behavior of the model in [17], namely the stability of periodic solutions, is explored as a function of the most important control parameters. The model includes: (1) human-like geometry; (2) energy absorption induced by ground-foot impact; and (3) control for stabilization. Mathematical tools for piecewise-smooth periodic systems are applied to demonstrate the feasibility of the model and to analyze qualitatively the global behavior of different types of running. More specifically, we present stable forward, zero velocity and backward hopping locomotion. We then perform an analysis of how the forward hopping locomotion and its stability depends on parameters and how it can be optimized in terms of locomotion velocity, apex height, energy efficiency and ground-foot impact intensity. Finally, going well beyond what has been reported in [17], we address biomechanical issues: the effect of tiptoe positioning on locomotion velocity and impact intensity.

\section{Motivation and background}

Important elements of our model and analysis are ground-foot interaction and stability control, which are both crucial for efficient running.

Depending on the form of running, the ground-foot interaction may lead to high-intensity impacts or collision. This may result in high kinetic energy absorption, which should be avoided in order to minimize the risk of injuries and also in order to increase energy efficiency during running $[5,6,9,18,19]$. There is a debate in the literature about the effect of running kinematics and foot placement patterns on the ground-foot collision intensity. For instance, Souza [20] states: "At this time, there is limited evidence that any foot strike pattern is more or less likely to cause a runner to sustain an injury". Another example is Jungers [18] who says "More studies ... are required to provide data instead of opinion, and testable models and scientific explanation instead of anecdotes". Therefore, a reliable mathematical model of legged locomotion could strongly contribute to the understanding of legged locomotion.

Despite considerable research efforts [21,22], the neural processes behind human balancing tasks are still not completely understood. An interesting approach is to compare the required mechanical energy consumption when standing still and during locomotion-when walking, running and hopping. Postural sway observed during standing still clearly demonstrates that certain 
energy input is required for the stabilization of the otherwise unstable upright standing position. Locomotion, on the other hand, is typically a periodic-like motion, which requires energy from the muscles to both overcome losses and to ensure stability, i.e., prevent falling. A long-term goal is to answer the question whether the energy required for stabilization is smaller in the case of locomotion compared to standing still.

\subsection{Hopping models and ground-foot collision}

Many aspects of periodic motion of legged systems were detailed in [15], where several walking, running and hopping models are reviewed. The classical SLIP model provides a fundamental background for hopping leg models. It represents the body inertia as a point mass which bounces along on a single elastic massless leg. The supporting role of the leg in each stance phase is characterized by the inverted pendulum. However, geometric features of a human leg and the groundfoot collision are not described by the SLIP model. The stable static configurations of a three-segmented leg model were presented in [2] for a case where the inertia and dynamic effects of the body segments are neglected and the body weight is modeled by a point mass located at the hip. Self-stable running-like locomotion for this model was presented in [16], showing that the stable domain in terms of landing angle and horizontal velocity is larger than for the SLIP model. Apart from the elimination of inertial forces, masslessness of the segments leads to smooth dynamics, i.e., there are no impulsive forces present when the foot comes into contact with the ground. This smoothness property allows one to use well-established analytical and numerical approaches for smooth system.

A mechanical model containing the foot and the shank was introduced in [19] for the investigation of the impulsive forces and the effect of the foot strike pattern. The foot-shank system falls vertically and the foot hits a fixed point; the contact point position is characterized by the strike index $s=l_{\mathrm{s}} / l$, where $l_{\mathrm{S}}$ indicates the location of the resultant force $\mathbf{F}_{\mathrm{p}}$ of foot pressure and $l$ is the foot length; see Fig. 1. An extension of the two-segmented model of [19] was introduced in [5] by involving a variable ankle angle and a horizontal velocity. Both [5] and [19] report that the effective mass and, therefore, the impact intensity is lower for forefoot-strike than for rear-foot-strike (heel-

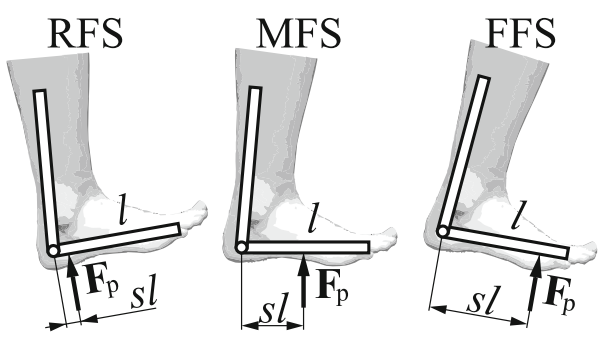

Fig. 1 Foot strike patterns identified by the index $s$ : rear-foot strike (RFS), mid-foot strike (MFS) and forefoot strike (FFS)

strike), when the strike index satisfies $s \in[0.7,1]$ and $s \in[0,0.3]$, respectively.

Results in [6] showed that, in addition to the strike pattern, the angle of the shank also strongly affects the properties of the ground-foot impact. The model in [6] involves the thigh and the total mass of the human body; the segments perform a rigid-body-like motion in the pre-impact phase with no relative motion of the body segments. However, this is not realistic and, in order to perform further biomechanical analysis of human running performance, we introduced a dynamic model with torque control in $[17,23]$ as an extension of the model from [16]. This model includes the masses of the segments so that the effects of the inertial forces can be determined. Moreover, it provides measures for the ground-foot collision, including kinetic energy absorption and effective mass, and it also provides a realistic pre-impact configuration.

The advantage of the presented numerical study over an experiment is that in the model the control parameters can be tuned to optimize different cost functions, such as running speed, energy efficiency and impact intensity. In this paper, we demonstrate the feasibility of this model by reporting results of a detailed analysis, including of the stability of different types of hopping motion; the mathematical details of the model are given in 3.1 .

\section{Dynamic model of the controlled hopping leg}

Hopping and running motion can be divided into flight (airborne) and ground (stance) phases, which are connected by ground-foot collisions [24]. For running and hopping robotic or biomechanical systems, some segments of the limbs periodically come into contact with the ground; therefore, the motion of these body segments are constrained during the duration of the stance 
phase. The intermittent constraints of the limb(s) lead to the absorption of a certain portion of the kinetic energy, referred to as constrained motion space kinetic energy (CMSKE) $[3,5,6]$. Since the energy loss of passive walkers [25] is caused mostly by the foot impact, CMSKE is often used as an energy efficiency indicator. It can also be used as an indicator of foot impact intensity, since it is proportional to the contact reaction force impulse and also to the peak reaction force $[5,26]$. During the stance phase, kinetic energy accumulates in the rest of the body while the muscles provide further mechanical power; finally, the ground-foot contact phase terminates.

The model used in the present study was developed to include and analyze the foot-ground impact described above. The body segments move downward before the end of the flight phase. The flight phase terminates when the tiptoe, denoted by point A in Fig. 2, comes into contact with the ground. During the ground phase, the active spring-damper system increases the total kinetic energy of the system. As a result, the height of the next hop is not lower than the height of the previous hop, in spite of the energy lost. The controller (detailed in Sect. 3.2) aims to maintain a nominal mechanical energy level $E_{0}$.

\subsection{Mechanical structure}

Figure 2 presents a diagram of the mechanics of the model. Segments 1, 2 and 3 correspond to the foot, shank and thigh, respectively. Similarly, the points A, $\mathrm{B}, \mathrm{C}$ and D correspond to the tiptoe, the ankle joint, the knee joint and the hip joint, respectively. The reaction wheel plays the role of the upper body, and the torque $M_{\mathrm{D}}$ is acting between the reaction wheel and the thigh. The mass of the reaction wheel and its moment of inertia with respect to the $y$-axis through point $\mathrm{D}$ are $m_{\mathrm{r}}$ and $J_{\mathrm{r}}$, respectively. The three homogeneous prismatic bars are characterized by their masses $m_{i}$ and lengths $l_{i}$, $i=1,2,3$; they are connected by torsional springs of stiffness $k_{\mathrm{B}}$ and $k_{\mathrm{C}}$. The overall center of mass (CoM) is located at point $\mathrm{G}$. Control torques are applied at joints B, C and D, as it is shown in the right panel of Fig. 2 . Actuating torques $M_{\mathrm{B}}$ and $M_{\mathrm{C}}$ implement the motion described by the control law, which is introduced below in Sect. 3.2.

The model has a total of 6 DoFs and the general coordinates are chosen to be $\mathbf{q}=\left[x_{\mathrm{A}}, z_{\mathrm{A}}, \theta_{1}, \theta_{12}, \theta_{23}, \theta_{\mathrm{r}}\right]^{\mathrm{T}}$,

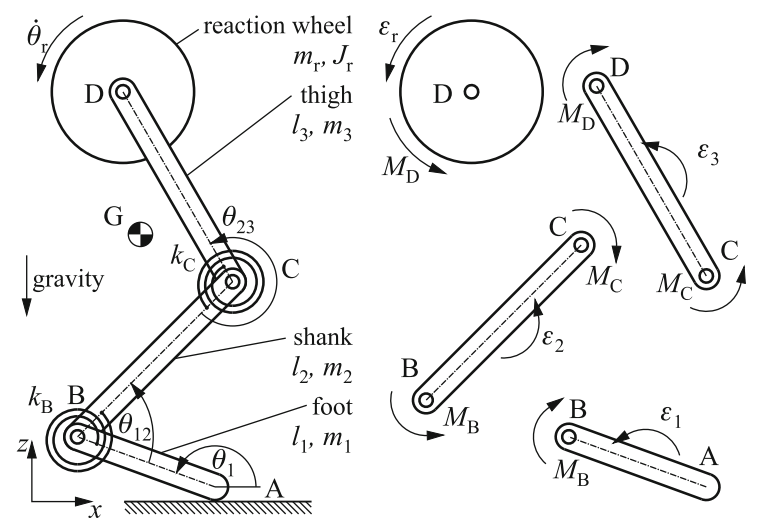

Fig. 2 Segmented leg model with torsional springs, under vertical gravity above a flat, rigid, horizontal ground (left). Freebody-diagram showing control torques (right)

where $x_{\mathrm{A}}$ and $z_{\mathrm{A}}$ are the Cartesian coordinates of the tiptoe, $\theta_{1}$ is the foot angle measured from the horizontal, $\theta_{12}$ and $\theta_{23}$ are the relative angles of the ankle and the knee, respectively, and $\theta_{\mathrm{r}}$ is the angle of the reaction wheel. The tiptoe is fixed to the ground during the ground phase.

The equation of motion assumes the general form:

$\mathbf{H}(\mathbf{q}) \ddot{\mathbf{q}}+\mathbf{C}(\mathbf{q}, \dot{\mathbf{q}})=\mathbf{Q}(\mathbf{q}, \dot{\mathbf{q}})$,

where $\dot{\mathbf{q}} \in \mathbb{R}^{n}$ and $\ddot{\mathbf{q}} \in \mathbb{R}^{n}$ are the general velocity and the acceleration, respectively, with $n=6$. The general mass matrix is denoted by $\mathbf{H} \in \mathbb{R}^{n \times n}$, and $\mathbf{C} \in \mathbb{R}^{n}$ represents the inertial force vector. The general force $\mathbf{Q} \in \mathbb{R}^{n}$ contains the gravitational forces, the torques of the torsional springs and the control torques.

Nonlinear spring characteristics due to the muscletendon dynamics, as mentioned in [2], is not considered here in order to keep the number of parameters manageable. The torques exerted by the linear torsional springs of stiffness $k_{\mathrm{B}}$ and $k_{\mathrm{C}}$ are given by

$k_{\mathrm{B}}\left(\theta_{12}-\alpha_{12}\right) \quad$ and $k_{\mathrm{C}}\left(\theta_{23}-\alpha_{23}\right)$,

where $\alpha_{12}$ and $\alpha_{23}$ denote the ankle and knee joint angles corresponding to the unstretched springs, respectively.

\subsection{Torque control}

The control law determines the required active torques in Eqs. (3)-(5) and (7)-(9) based on the motion of the segments and information about the actual phase. Control concepts based on trajectory tracking and those 
based on feedforward inputs form the central pattern generators are clearly distinguished and described in greater detail in [4] and in [15]. Our control approach rather fits into the latter category in that we consider a type of feedback for the control; the strategy of keeping the leg segments on some prescribed trajectories is not employed as part of the work presented here.

In the flight phase, the control torques $M_{\mathrm{B}}$ and $M_{\mathrm{C}}$ behave as damping torques and suppress the possible oscillations of the relative segmental angles as (3) and (4) show. The horizontal position $x_{\mathrm{A}}$ of the tiptoe relative to horizontal position $x_{\mathrm{G}}$ of the CoM has a large effect on stability, on locomotion velocity $\bar{v}$ and on impact intensity. Approaching and reaching the nominal horizontal position of point $\mathrm{A}$ is facilitated by a proportional-derivative (PD) controller defined in (5) below. We consider this as the foot touchdown preparation. The torques $M_{\mathrm{B}}^{\mathrm{F}}, M_{\mathrm{C}}^{\mathrm{F}}$ and $M_{\mathrm{D}}^{\mathrm{F}}$ are given by

$M_{\mathrm{B}}^{\mathrm{F}}=-D_{\mathrm{B}} \dot{\theta}_{12}$,

$M_{\mathrm{C}}^{\mathrm{F}}=-D_{\mathrm{C}} \dot{\theta}_{23}$,

$M_{\mathrm{D}}^{\mathrm{F}}=P\left(x_{\mathrm{A}}-\left(x_{\mathrm{G}}+x_{\Delta}\right)\right)+D\left(\dot{x}_{\mathrm{A}}-\dot{x}_{\mathrm{G}}\right)$,

where $D_{\mathrm{B}}, D_{\mathrm{C}}, P$ and $D$ are tuned control gains. The nominal tiptoe position is modified by

$x_{\Delta}=P_{\Pi} \Pi_{\mathrm{A}}-K_{v}$,

where $\Pi_{\mathrm{A}}$ is the angular momentum about the point $\mathrm{A}$, $P_{\Pi}$ is an associated control gain and $K_{v}$ is a control parameter responsible for tuning the nominal locomotion speed $\bar{v}$.

During the ground phase, CMSKE is recovered by means of the control torques (7) and (8) exerted at the ankle and the knee. The goal is to keep the total mechanical energy $E$ at the freely chosen target energy level $E_{0}$. The mechanical power of these torques is positive only if the joints are in extension, so that $\dot{\theta}_{12}$ is positive and $\dot{\theta}_{23}$ is negative. The torque $M_{\mathrm{D}}^{\mathrm{G}}$ in (9) prevents the continuous growth of the angular velocity $\dot{\theta}_{\mathrm{r}}$ of the reaction wheel. The control torques $M_{\mathrm{B}}^{\mathrm{G}}, M_{\mathrm{C}}^{\mathrm{G}}$ and $M_{\mathrm{D}}^{\mathrm{G}}$ are given by

$M_{\mathrm{B}}^{\mathrm{G}}=P_{E}\left(E-E_{0}\right) \operatorname{sgn}\left(\dot{\theta}_{12}\right)$,

$M_{\mathrm{C}}^{\mathrm{G}}=P_{E}\left(E-E_{0}\right) \operatorname{sgn}\left(-\dot{\theta}_{23}\right)$,

$M_{\mathrm{D}}^{\mathrm{G}}=-P_{\mathrm{r}} \theta_{\mathrm{r}}-D_{\mathrm{r}} \dot{\theta}_{\mathrm{r}}$,

where $P_{E}, P_{\mathrm{r}}$ and $D_{\mathrm{r}}$ are control gains.
3.3 Flight and ground phase transitions

The system is described by the state variable vector $\mathbf{x}=[\mathbf{q}, \dot{\mathbf{q}}]^{\mathrm{T}}$, where $\mathbf{x} \in \mathbb{R}^{2 n}$. The system dynamics is given by

$\dot{\mathbf{x}}(t)=\mathbf{f}_{\mathrm{F}}(\mathbf{x}(t))$ in flight phase,

$\dot{\mathbf{x}}(t)=\mathbf{f}_{\mathrm{G}}(\mathbf{x}(t))$ in ground phase,

where

$\mathbf{f}_{\mathrm{F}}=\left[\begin{array}{c}\dot{\mathbf{q}} \\ -\mathbf{H}^{-1}(\mathbf{C}-\mathbf{Q})\end{array}\right]$ and

$\mathbf{f}_{\mathrm{G}}=\left[\begin{array}{c}\mathbf{0}_{2 \times 1} \\ \dot{\mathbf{q}}_{\mathrm{red}} \\ \mathbf{0}_{2 \times 1} \\ -\mathbf{H}_{\mathrm{red}}^{-1}\left(\mathbf{C}_{\mathrm{red}}-\mathbf{Q}_{\mathrm{red}}\right)\end{array}\right]$

are smooth vector fields that describe the flight and the ground phase, respectively. Here, $\dot{\mathbf{q}}_{\mathrm{red}}=\left[\dot{\theta}_{1}, \dot{\theta}_{12}\right.$, $\left.\dot{\theta}_{23}, \dot{\theta}_{\mathrm{r}}\right]^{\mathrm{T}}$ is a reduced general velocity vector; similarly, the reduced mass matrix $\mathbf{H}_{\text {red }}$ is obtained by truncating the first two rows and columns of $\mathbf{H}$, while $\mathbf{C}_{\text {red }}$ and $\mathbf{Q}_{\text {red }}$ are obtained by truncating the first two rows of $\mathbf{C}$ and $\mathbf{Q}$, respectively. Equation (13) in effect imposes $\ddot{x}_{\mathrm{A}}=0$ and $\ddot{z}_{\mathrm{A}}=0$ for the tiptoe coordinates.

The overall system features switching and discontinuities of the solutions due to the transitions between the two phases of motion, which also need to be defined to determine the dynamics; one speaks of a hybrid system $[3,27,28]$. The transition between (10) and (11) occurs at codimension-one switching (hyper) surfaces $\Sigma_{\mathrm{G} 2 \mathrm{~F}}$ and $\Sigma_{\mathrm{G} 2 \mathrm{~F}}$; these are illustrated in Fig. 3.

\subsubsection{Flight to ground}

The flight phase is terminated by the foot touchdown. In the presented model, the ground contact is modeled as a single point contact at point $\mathrm{A}$. The contact force at any other body segments is not considered. In certain parameter regions, this leads to stable hopping solutions while point $\mathrm{B}$ penetrates into the ground, which is physically unfeasible. This modeling issue arises commonly in single contact point models, see, e.g., $[5,19]$. Physical feasibility is monitored, and unfeasible solutions are detected in Sect. 4.

The flight to ground (F2G) transition occurs when $\Sigma_{\mathrm{F} 2 \mathrm{G}}$, defined by

$h_{\mathrm{F} 2 \mathrm{G}}(\mathbf{x}):=z_{\mathrm{A}}=0$,

is reached. The F2G transition involves an impact, which corresponds to a discontinuity of the solution. 


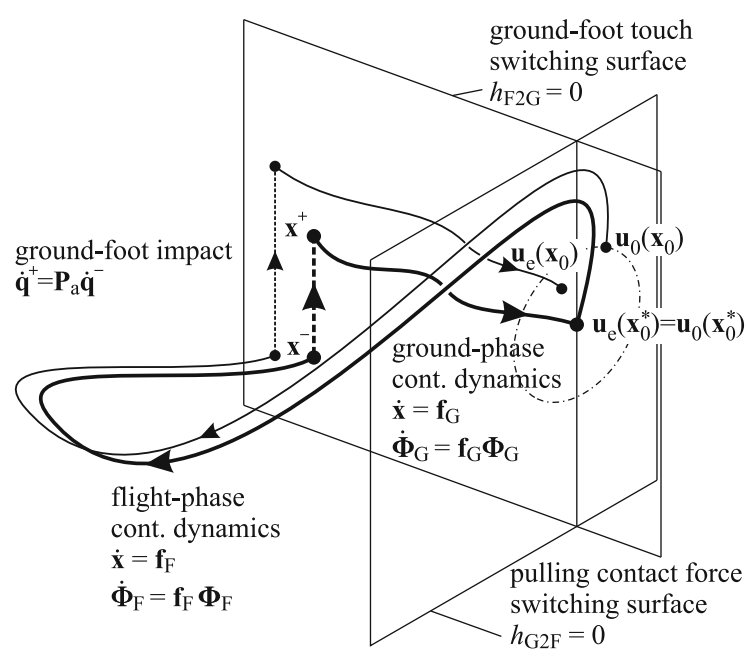

Fig. 3 Schematic picture of the overall system, showing a stable relative period and a nearby trajectory with flight phase, groundfoot impact, ground phase and ground-foot detachment

We assume that the ground-foot collision is instantaneous and completely inelastic so that there is no rebound $[5,6,19]$. Furthermore, we assume that the friction coefficient is large enough so that the foot does not slip on the ground; this means that it satisfies

$\mu_{\mathrm{C}} \geq\left|\frac{\boldsymbol{\gamma}_{\mathbf{q}, \mathrm{n}} \mathbf{H}^{-1} \boldsymbol{\gamma}_{\mathbf{q}, \mathrm{t}}^{\mathrm{T}}}{\boldsymbol{\gamma}_{\mathbf{q}, \mathrm{n}} \mathbf{H}^{-1} \boldsymbol{\gamma}_{\mathbf{q}, \mathrm{n}}^{\mathrm{T}}}\right|$,

where $\boldsymbol{\gamma}_{\mathbf{q}, \mathrm{n}}$ and $\boldsymbol{\gamma}_{\mathbf{q}, \mathrm{t}}$ are the gradients of the constraints in the normal and tangential directions, respectively.

Under these assumptions, the tiptoe remains constrained to a single point on the ground during the ground phase, as in $[5,6,17,23,29]$. Consequently, the velocities $\dot{x}_{\mathrm{A}}$ and $\dot{z}_{\mathrm{A}}$ become zero and the angular velocities change abruptly when the solution reaches $\Sigma_{\mathrm{F} 2 \mathrm{G}}$. The jump function

$\mathbf{g}_{\mathrm{F} 2 \mathrm{G}}(\mathbf{x})=\left[\begin{array}{c}\mathbf{q} \\ \left(\mathbf{I}_{n \times n}-\mathbf{P}_{\mathrm{c}}\right) \dot{\mathbf{q}}\end{array}\right]$

maps the state to the specified location [30], by projecting the velocities into the admissible direction of the constraints $\boldsymbol{\gamma}(\mathbf{q})[6,31,32]$. Here

$\mathbf{P}_{\mathrm{c}}=\mathbf{H}^{-1} \boldsymbol{\gamma}_{\mathbf{q}}^{\mathrm{T}}\left(\boldsymbol{\gamma}_{\mathbf{q}} \mathbf{H}^{-1} \boldsymbol{\gamma}_{\mathbf{q}}^{\mathrm{T}}\right)^{-1} \boldsymbol{\gamma}_{\mathbf{q}}$

is the projection matrix onto the subspace of constrained motion.

The instantaneous ground-foot collision leads to infinitely large instantaneous forces, over infinitesimal time duration, so that the net impulse due to the impact force is finite $[5,19]$. Since CMSKE is proportional to the impulse of the contact reaction force and also to the peak reaction force [5,26], it is used for characterizing the foot impact intensity in this work.

CMSKE, which vanishes at foot touchdown, is calculated with the pre-impact general velocity as in [26] as

$T_{\mathrm{c}}=\frac{1}{2} \dot{\mathbf{q}}^{\mathrm{T}} \mathbf{P}_{\mathrm{c}}^{\mathrm{T}} \mathbf{H} \mathbf{P}_{\mathrm{c}} \dot{\mathbf{q}}$.

The papers [5] and [26] showed that foot strike intensity can be characterized by the CMSKE, which depends on the pre-impact configuration and velocity, and the effective mass matrix $\mathbf{H}_{\mathrm{e}}=\mathbf{P}_{\mathrm{c}}^{\mathrm{T}} \mathbf{H} \mathbf{P}_{\mathrm{c}}$. The effective mass concept for foot impact is introduced in [33] for a one DoF model of legged locomotion.

\subsubsection{Ground to flight}

The ground to flight (G2F) transition at the surface $\Sigma_{\mathrm{G} 2 \mathrm{~F}}$, defined by

$h_{\mathrm{G} 2 \mathrm{~F}}(\mathbf{x}):=\lambda_{z}=0$,

is a switch from (11) to (10) without a discontinuity of the solution. Here, the Lagrange multiplier $\lambda_{z}$ is the vertical ground contact force acting on the tiptoe, and a negative and positive sign of $\lambda_{z}$ refers to a pushing and a pulling force, respectively. The contact force is calculated by solving the linear algebraic system

$\left[\begin{array}{c}\ddot{\mathbf{q}} \\ \lambda\end{array}\right]=\left[\begin{array}{cc}\mathbf{H}(\mathbf{q}) & \boldsymbol{\gamma}_{\mathbf{q}}^{\mathrm{T}}(\mathbf{q}) \\ \boldsymbol{\gamma}_{\mathbf{q}}(\mathbf{q}) & \mathbf{0}\end{array}\right]\left[\begin{array}{c}\mathbf{Q}(\mathbf{q}, \dot{\mathbf{q}})-\mathbf{C}(\mathbf{q}, \dot{\mathbf{q}}) \\ -\dot{\boldsymbol{\gamma}}_{\mathbf{q}}(\mathbf{q}, \dot{\mathbf{q}}) \dot{\mathbf{q}}\end{array}\right]$

adopted from [31]. The second time derivative $\ddot{\gamma}=$ $\boldsymbol{\gamma}_{\mathrm{q}} \ddot{\mathbf{q}}+\dot{\boldsymbol{\gamma}}_{\mathbf{q}} \dot{\mathbf{q}}$ of the geometric constraint vector $\boldsymbol{\gamma}(\mathbf{q})$ appears in (20), where

$\boldsymbol{\gamma}_{\mathbf{q}}(\mathbf{q})=\nabla_{\mathbf{q}} \boldsymbol{\gamma}(\mathbf{q})$

is the gradient of the constraint. The ground-foot contact is represented by $\boldsymbol{\gamma}(\mathbf{q})=\mathbf{0}$ with

$\boldsymbol{\gamma}(\mathbf{q})=\left[x_{\mathrm{A}}, z_{\mathrm{A}}\right]^{\mathrm{T}}$.

Control torques included in $\mathbf{Q}(\mathbf{q}, \dot{\mathbf{q}})$ in (20) are calculated with the control law (7)-(9) in the ground phase.

\subsection{Finding stable relative periodic orbits}

Our focus is on hopping motion, which corresponds to a relative periodic orbit with a translational symmetry in the lateral ground direction, given by the variable $x$. Such an orbit is shown in the phase space sketch in Fig. 3. 
A relative periodic orbit is found with a shooting approach as a root $\mathbf{x}_{0}^{*}$ of

$\mathbf{F}\left(\mathbf{x}_{0}\right):=\mathbf{u}_{\mathrm{e}}\left(\mathbf{x}_{0}\right)-\mathbf{u}_{0}\left(\mathbf{x}_{0}\right)$,

where $\mathbf{u}_{0}\left(\mathbf{x}_{0}\right)$ is a subvector of a point $\mathbf{x}_{0} \in \Sigma_{\mathrm{F} 2 \mathrm{G}}$ and $\mathbf{u}_{\mathrm{e}}\left(\mathbf{x}_{0}\right)$ is the corresponding subvector at the end point of the solution trajectory of the overall system that starts at $\mathbf{x}_{0}$ and ends again in the section $\Sigma_{\mathrm{F} 2 \mathrm{G}}$ by reaching $\Sigma_{\mathrm{G} 2 \mathrm{~F}}$. We remark that $\mathbf{u}_{0}$ has lower dimension than $\mathbf{x}_{0}$, because some of the state variables (both coordinates and velocities) are known when the tiptoe is in contact with the ground. This reduction in the number of unknowns considerably reduces the computation time.

\subsubsection{Monodromy matrix and Floquet multipliers}

To determine the stability of the relative periodic orbit given by $\mathbf{x}_{0}^{*}$ (see Fig. 3), we proceed as described in $[3,30,34]$. During the flight and ground phases we integrate from $t=0$ for the two vector fields $\mathbf{f}_{\mathrm{F}}$ and $\mathbf{f}_{\mathrm{G}}$, respectively, the first variational equation

$$
\begin{aligned}
\dot{\mathbf{x}}(t) & =\mathbf{f}(\mathbf{x}(t)) ; & \mathbf{x}\left(t_{\text {init }}\right)=\mathbf{x}_{0}^{*}, \\
\dot{\boldsymbol{\Phi}}(t) & =\mathbf{f}_{\mathbf{x}}(\mathbf{x}(t)) \boldsymbol{\Phi}(t) ; & \boldsymbol{\Phi}\left(t_{\text {init }}\right)=\mathbf{I}_{2 n \times 2 n},
\end{aligned}
$$

where $\mathbf{I}$ is $2 n \times 2 n$ identity matrix and $\mathbf{f}_{\mathbf{x}}$ is Jacobian of the vector field. This results in the fundamental solution matrices $\boldsymbol{\Phi}_{\mathrm{F}}(t)$ and $\boldsymbol{\Phi}_{\mathrm{G}}(t)$ [34].

The Jacobian or monodromy matrix $\tilde{\boldsymbol{\Phi}}(t)$ over the entire relative periodic orbit is then obtained as the composition

$$
\tilde{\boldsymbol{\Phi}}\left(t_{\mathrm{G} 2 \mathrm{~F}}\right)=\mathbf{S}_{\mathrm{G} 2 \mathrm{~F}} \boldsymbol{\Phi}_{\mathrm{G}}\left(t_{\mathrm{G} 2 \mathrm{~F}}\right) \mathbf{S}_{\mathrm{F} 2 \mathrm{G}} \boldsymbol{\Phi}_{\mathrm{F}}\left(t_{\mathrm{F} 2 \mathrm{G}}\right) .
$$

Here, $\mathbf{S}_{\mathrm{G} 2 \mathrm{~F}}$ and $\mathbf{S}_{\mathrm{F} 2 \mathrm{G}}$ describe the contribution of the mappings at the switchings, which are known as the saltation matrices and are related to the Jacobian of the transition condition [35,36]. A saltation matrix can be determined $[3,30]$ as

$\mathbf{S}=\mathbf{g}_{\mathbf{x}}^{-}+\frac{\left(\mathbf{f}^{+}-\mathbf{g}_{\mathbf{x}}^{-} \mathbf{f}^{-}\right) h_{\mathbf{x}}^{-}}{h_{\mathbf{x}}^{-} \mathbf{f}^{-}}$,

where $\mathbf{g}_{\mathbf{x}}^{-}$is the gradient of the jump function, and $h_{\mathbf{x}}^{-}$ is the gradient of the indicator function [see (14) and (19)] evaluated at the state just before the transition. For the G2F transition, $\mathbf{S}_{\mathrm{G} 2 \mathrm{~F}}$ is obtained from (27) by applying the substitutions

$$
\begin{aligned}
& \mathbf{f}^{+}=\mathbf{f}_{\mathrm{F}}\left(\mathbf{x}_{\mathrm{G} 2 \mathrm{~F}}\right), \quad \mathbf{f}^{-}=\mathbf{f}_{\mathrm{G}}\left(\mathbf{x}_{\mathrm{G} 2 \mathrm{~F}}\right), \\
& \mathbf{g}_{\mathbf{x}}^{-}=\mathbf{I}_{2 n \times 2 n}, \quad h_{\mathbf{x}}^{-}=\nabla_{\mathbf{x}} h_{\mathrm{G} 2 \mathrm{~F}}\left(\mathbf{x}_{\mathrm{G} 2 \mathrm{~F}}\right) .
\end{aligned}
$$

For the $\mathrm{F} 2 \mathrm{G}$ transition, the discontinuity mapping $\mathbf{g}_{\mathrm{F} 2 \mathrm{G}}$ (16) is taken into account, and substitutions

$\mathbf{f}^{+}=\mathbf{f}_{\mathrm{G}}\left(\mathbf{x}_{\mathrm{F} 2 \mathrm{G}}^{+}\right), \quad \mathbf{f}^{-}=\mathbf{f}_{\mathrm{F}}\left(\mathbf{x}_{\mathrm{F} 2 \mathrm{G}}^{-}\right)$,

$\mathbf{g}_{\mathbf{x}}^{-}=\nabla_{\mathbf{x}} \mathbf{g}_{\mathrm{F} 2 \mathrm{G}}\left(\mathbf{x}_{\mathrm{F} 2 \mathrm{G}}^{-}\right), \quad h_{\mathbf{x}}^{-}=\nabla_{\mathbf{x}} h_{\mathrm{F} 2 \mathrm{G}}\left(\mathbf{x}_{\mathrm{F} 2 \mathrm{G}}^{-}\right)$

are applied for the calculation of $\mathbf{S}_{\mathrm{F} 2 \mathrm{G}}$. Here, $\mathbf{x}_{\mathrm{F} 2 \mathrm{G}}^{-}$ and $\mathbf{x}_{\mathrm{F} 2 \mathrm{G}}^{+}=\mathbf{g}_{\mathrm{F} 2 \mathrm{G}}\left(\mathbf{x}_{\mathrm{F} 2 \mathrm{G}}^{-}\right)$are, respectively, the state just before and just after the impacting transition (see Fig. 3).

The stability of the relative periodic orbit is determined from the eigenvalues of the monodromy matrix $\tilde{\boldsymbol{\Phi}}\left(t_{\mathrm{G} 2 \mathrm{~F}}\right)$, which are known as the Floquet multipliers [34]. There is always a Floquet multiplier equal to 1, because of periodicity; moreover, due to the translation in the $x$-direction there is a second Floquet multiplier 1. The relative periodic orbit is stable when all other Floquet multipliers have modulus less than 1 .

\subsubsection{Tuning control parameters}

Tuning of the physical and control parameters is crucial for the study of the model. Our goal here is to find the control parameter set associated with a stable periodic motion for which some cost function, such as energy use, is minimized. We collect 10 parameters of (3)-(9) in the vector $\mathbf{p}$

$$
\begin{aligned}
\mathbf{p}= & {\left[\alpha_{12}, \alpha_{23}, D_{\mathrm{B}}, D_{\mathrm{C}}, P, D,\right.} \\
& \left.P_{\Pi}, K_{v}, P_{E}, E_{0}, P_{\mathrm{r}}, D_{\mathrm{r}}\right] .
\end{aligned}
$$

An initial parameter set $\mathbf{p}$, which provides stable hopping, was found using a trial-and-error method as reported in [17]. To tune the parameters $\mathbf{p}$, the associated solution $\mathbf{x}_{0}^{*}$ of (23) was found by continuation for a parameter sweep during which minima of the given cost function were detected. Note that if the cost function is chosen as the norm of the largest eigenvalue of $\tilde{\boldsymbol{\Phi}}$, then it characterizes stability. Another possible choice is to use CMSKE [denoted $T_{\mathrm{c}}$ in (18)] as the cost function, which characterizes impact intensity and energy efficiency.

\section{Results on stable locomotion}

We consider now the test-case example of a 24-yearold male subject with bodyweight of $73 \mathrm{~kg}$ and body height of $173.1 \mathrm{~cm}$, adopted from [37] with further anatomical data as given in Table 1. The parameters of the torsional springs at the joints are independent of human 
Table 1 Inertial and geometric data of the body segments

\begin{tabular}{llll}
\hline & Mass $(\mathrm{kg})$ & Length/ height $(\mathrm{m})$ & $\begin{array}{l}\text { Mass moment of inertia w.r.t. } \\
\text { CoM }\left(\mathrm{kg} \mathrm{m}^{2}\right)\end{array}$ \\
\hline Body & $m_{\mathrm{b}}=73$ & $l_{\mathrm{b}}=1.731$ & - \\
Feet & $0.0274 m_{\mathrm{b}}$ & $0.0885 l_{\mathrm{b}}$ & $1 / 12 m_{1} l_{1}^{2}$ \\
Shanks & $0.0866 m_{\mathrm{b}}$ & $0.2470 l_{\mathrm{b}}$ & $1 / 12 m_{2} l_{2}^{2}$ \\
Thighs & $0.2832 m_{\mathrm{b}}$ & $0.2320 l_{\mathrm{b}}$ & $1 / 12 m_{3} l_{3}^{2}$ \\
Trunk & $0.6028 m_{\mathrm{b}}$ & - & 1.978 \\
\hline
\end{tabular}

Table 2 Parameters of the torsional springs placed at the ankle and the knee joints

\begin{tabular}{lr}
\hline$\alpha_{12}\left(^{\circ}\right)$ & 80 \\
$\alpha_{23}\left({ }^{\circ}\right)$ & 225 \\
$k_{\mathrm{B}}(\mathrm{Nm})$ & 1200 \\
$k_{\mathrm{C}}(\mathrm{Nm})$ & 1300 \\
\hline
\end{tabular}

Table 3 Control parameters: $E_{0}$ and $K_{v}$ are different for the different cases $\mathbf{A}, \mathbf{B}$ and $\mathbf{C}$, all the other control parameters are fixed

\begin{tabular}{llll}
\hline Case & A & B & C \\
\hline$K_{v}(\mathrm{~m})$ & 0.4 & 0 & -0.4 \\
$E_{0}(\mathrm{~J})$ & 1000 & & \\
$D_{\mathrm{B}}(\mathrm{Nms})$ & 15 & & \\
$D_{\mathrm{C}}(\mathrm{Nms})$ & 20 & & \\
$P(\mathrm{~N})$ & 950 & & \\
$D(\mathrm{Ns})$ & 50 & & \\
$P_{\Pi}[1 /(\mathrm{Ns})]$ & 0.01 & \\
$P_{E}[1]$ & 0.2 & \\
$P_{\mathrm{r}}(\mathrm{Nm})$ & 4 & \\
$D_{\mathrm{r}}(\mathrm{Nms})$ & 15 & \\
\hline
\end{tabular}

anthropometry and are shown in Table 2. The control parameters used in the study presented here are given in Table 3.

For these choices of parameters, relative periodic motion (i.e., hopping) was found. In what follows, the parameters $E_{0}$ and $K_{v}$ are considered as the main parameters, because they are responsible for the tuning of the apex height $z_{\mathrm{A} \text { max }}$ and the average horizontal locomotion velocity $\bar{v}$ of the hopping motion, respectively. The rest of the parameters remain fixed.
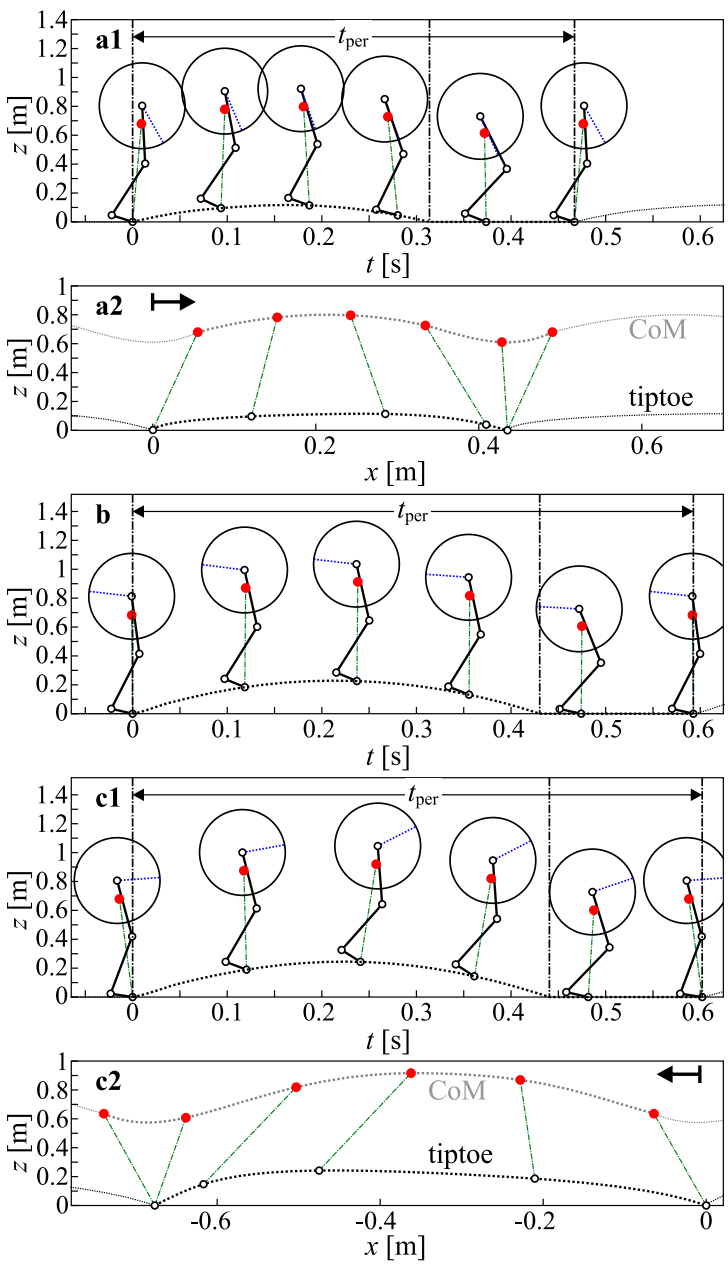

Fig. 4 Stable hopping motion for cases $\mathbf{A}, \mathbf{B}$ and $\mathbf{C}$ for $K_{v}=0.4$, $K_{v}=0$ and $K_{v}=-0.4$, respectively. Panels a1, b and $\mathrm{c} 1$ : instances of leg position over one period with tiptoe elevation $z_{\mathrm{A}}$ (dashed black curves), CoM position (red dots), line between the tiptoe and the CoM (green dash-dotted line), and angular position of the reaction wheel (blue doted lines). Panels a 2 and c2: Corresponding tiptoe path (black) and CoM path (gray dotted) in the $\left(x_{\mathrm{G}}, z_{\mathrm{G}}\right)$-plane for $\mathbf{A}$ and $\mathbf{C}$; the direction of locomotion is indicated by the arrow. (Color figure online) 


\subsection{Illustrative examples of stable hopping motion}

The model may display stable forward hopping, stable hopping motion on the spot and stable backward hopping, with a locomotion velocity that depends on the choice of $K_{v}$. Setting $K_{v}$ to $0.4,0$ and -0.4 , respectively, gives the hopping motions denoted $\mathbf{A}, \mathbf{B}$ and $\mathbf{C}$ and are illustrated in Fig. 4 over one complete period of the relative periodic orbit. Panels a1 and a2 for case A show forward locomotion, panel $b$ for case $\mathbf{B}$ shows hopping in one place, and panels $\mathrm{c} 1$ and $\mathrm{c} 2$ for case $\mathbf{C}$ show backward hopping locomotion. Here the time history of the tiptoe elevation is plotted together with the stroboscopic view of the model in panels $\mathrm{a} 1, \mathrm{~b}$ and $\mathrm{c} 1$. The tilt angle of the line between the tiptoe and the CoM at the beginning of the period is in correlation with the direction of locomotion: tilting to the right yields forward locomotion; no tilting yields hopping on the spot; and tilting to the left yields backward locomotion. The paths of the tiptoe and the CoM are shown in panels a2 and $\mathrm{c} 2$ for the forward and backward locomotion, respectively. We remark that tiptoe and the CoM paths are qualitatively similar to that of the SLIP model in [15].

Since forward locomotion is the most relevant from a practical point of view, we focus on case example $\mathbf{A}$ in the rest of the section. It is illustrated in more detail in Fig. 5. Panel a shows the relative periodic solution in projection onto the subspace $\left(z_{\mathrm{A}}, \theta_{\mathrm{r}}, \dot{z}_{\mathrm{A}}\right)$. It starts at the point G2F, which is located at the intersection of the switching surfaces $\Sigma_{\mathrm{G} 2 \mathrm{~F}}$ and $\Sigma_{\mathrm{F} 2 \mathrm{G}}$. After the flight phase, the solution has a jump within $\Sigma_{\mathrm{F} 2 \mathrm{G}}$. Finally, the ground phase stays on $\Sigma_{\mathrm{F} 2 \mathrm{G}}$ until it reaches $\Sigma_{\mathrm{G} 2 \mathrm{~F}}$ again. Different planar projections of the phase space are shown in Fig. 5b. As can be seen, all of the general coordinates are periodic in time, except for $x_{\mathrm{A}}$, which illustrates that the corresponding motion is indeed represented by a relative periodic orbit. Also note that the F2G transition generates a jump in each general coordinate, except in $\theta_{\mathrm{r}}$. The reason for the continuous solution for $\theta_{\mathrm{r}}$ is the lack of its inertial coupling with the other general coordinates.

Figure 6 shows, in panels a and $b$, time evolutions of the kinetic energy $T$, the potential function $U$ and the total mechanical energy $T+U$. The constrained motion space kinetic energy (CMSKE) $T_{\mathrm{c}}$ is indicated in both cases; this portion of the kinetic energy is absorbed during the F2G transition and then compensated for during the ground phase.

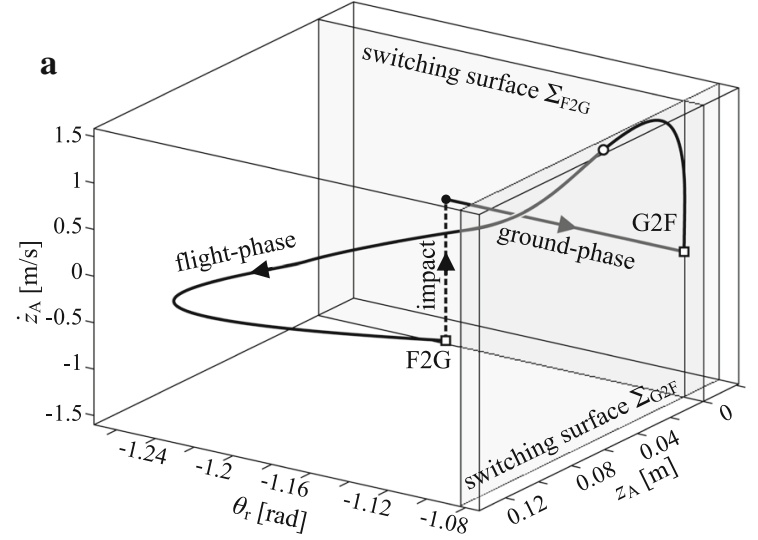

b
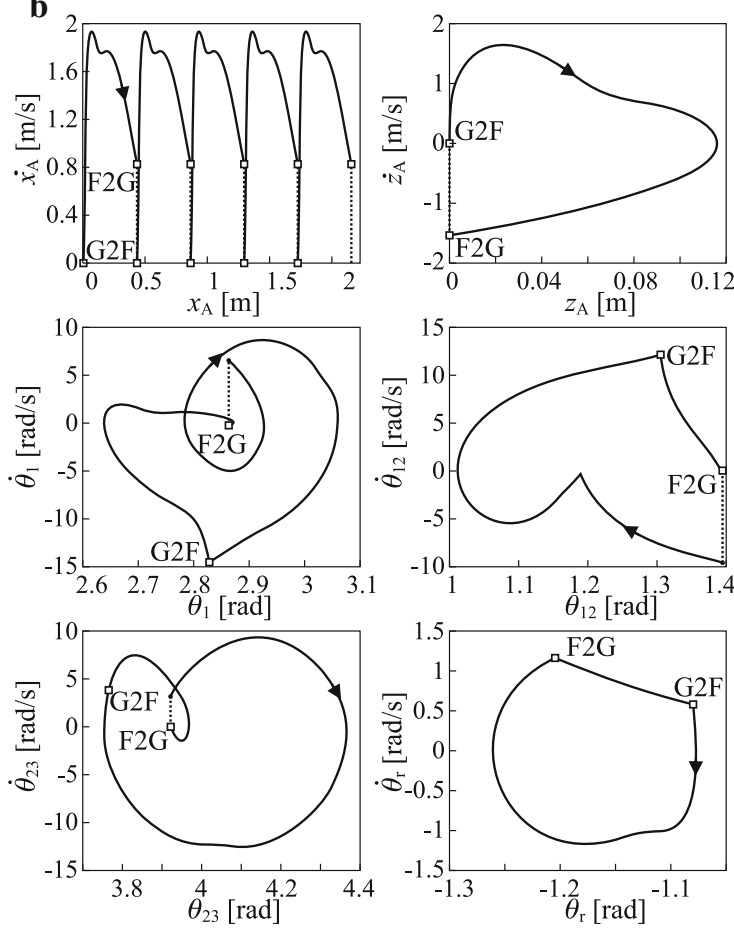

Fig. 5 Relative periodic orbit for case example $\mathbf{A}$, where the transitions $\mathrm{G} 2 \mathrm{~F}$ and $\mathrm{F} 2 \mathrm{G}$ are indicated by squares and the dotted lines indicate the discrete mapping after foot touchdown. a Shown in projection onto the subspace $\left(z_{\mathrm{A}}, \theta_{\mathrm{r}}, \dot{z}_{\mathrm{A}}\right)$. b Phase plane sections for each general coordinate

\subsection{Stability region and effect of main control parameters}

We now ascertain the role of the two main parameters $K_{v}$ and $E_{0}$. First of all, starting from the three known solutions $\mathbf{A}, \mathbf{B}$ and $\mathbf{C}$, we continue the relative periodic orbit in $K_{v}$ in the positive and the negative direc- 


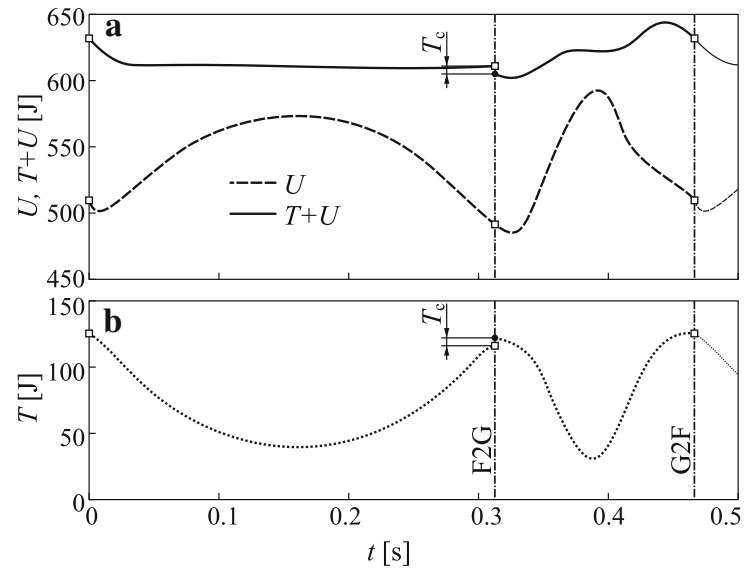

Fig. 6 Time evolution of kinetic and potential energy for case example $\mathbf{A}$, where a complete period goes from $t=0$ to the dash-dotted line G2F. The absorbed amount of kinetic energy (CMSKE) is indicated by $T_{\mathrm{c}}$

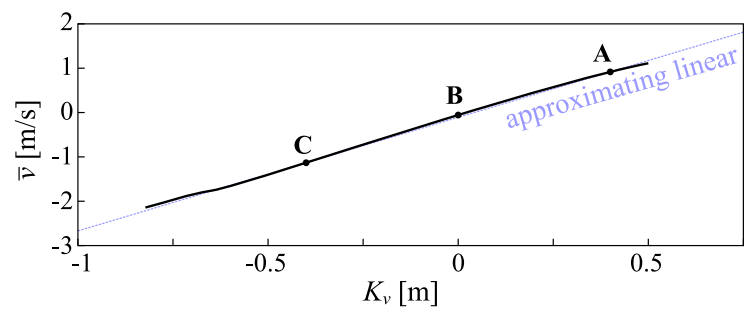

Fig. 7 Dependence of locomotion velocity $\bar{v}$ on control parameter $K_{v}$ for case example $\mathbf{A}$ (where $E_{0}=1000 \mathrm{~J}$ )

tion until the boundary of stability is reached. Figure 7 shows the induced locomotion velocity $\bar{v}$ as a function of $K_{v}$, which is approximately a linear function of $K_{v}$ for this particular value of $E_{0}$. Consequently, the locomotion velocity can be predicted by simple linear extrapolation.

The parameter $E_{0}$ is responsible for the tuning of the apex height of hopping, and the $K_{v}$-range of stability can be computed for different values of $E_{0}$. In this way, the stable region in the $\left(K_{v}, E_{0}\right)$-plane in Fig. 8 is determined, where parameter points for case examples $\mathbf{A}, \mathbf{B}$ and $\mathbf{C}$ are also indicated. Moreover, the continuation shown in Fig. 7 is indicated in Fig. 8 by the vertical dashed line at $E_{0}=1000 \mathrm{~J}$. We have already shown that the locomotion velocity is zero for case example $\mathbf{B}$. The locus of zero locomotion velocity can be computed as well, and it is shown in Fig. 8, where it divides the region of stability in subregions of forward and back-

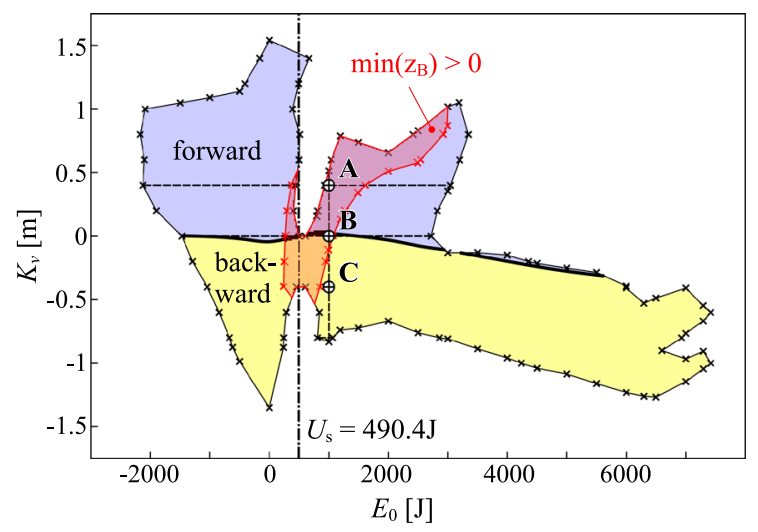

Fig. 8 Stability region in the $\left(E_{0}, K_{v}\right)$-plane. The black crosses indicate the points where the stability border was identified numerically. Case examples $\mathbf{A}, \mathbf{B}$ and $\mathbf{C}$ are indicated. The locus of zero velocity locomotion (thick black curve) separates the region of stable forward motion (light blue) from that of stable backward motion (light yellow). The energy level of standing still is indicated by the vertical dash-dotted line. The horizontal dashed lines indicate when $K_{v}=0 \mathrm{~m}$ and $K_{v}=0.4 \mathrm{~m}$. The physically feasible region, where the heel (point B) does not go below the surface level, is bounded by the red curves and indicated by opaque red shading. (Color figure online)

ward hopping. Notice also the narrowing of the stable region at about a nominal energy level of $E_{0}=500 \mathrm{~J}$. This happens near the dash-dotted line in Fig. 8 indicating the potential energy level $U_{\mathrm{s}}=490.4 \mathrm{~J}$ of standing still (ankle and knee angles are $\alpha_{12}$ and $\alpha_{23}$, respectively). This energy level is important, because a lower energy level cannot be reached permanently. On the other hand, the controller is able to ensure stable operation for a target energy level of $E_{0}<U_{\mathrm{s}}$ as well.

In the region highlighted with red color in Fig. 8, the ankle point $\mathrm{B}$ of the mechanical model shown in Fig. 2 does not touch the ground and the minimum of the vertical position $z_{\mathrm{B}}$ is larger than zero. Hence, the corresponding solutions are physically feasible. As is the case for the entire diagram in Fig. 8, size and shape of this feasibility region depend on the parameters of the model. Note that case examples $\mathbf{A}$ and $\mathbf{B}$ are physically feasible for the chosen parameter values.

Figure 9 shows the properties of the locomotion as a function of $E_{0}$, which were derived from continuation in $E_{0}$ from case $\mathbf{A}$ of forward locomotion and from case B of hopping on the spot. Panel a shows that the apex height $z_{\mathrm{A} \text { max }}$ of the hopping motion is not only affected by the nominal energy level $E_{0}$, but also by $K_{v}$. For $E_{0}>U_{\mathrm{s}}$, the apex height $z_{\mathrm{A} \max }$ can be approximated 

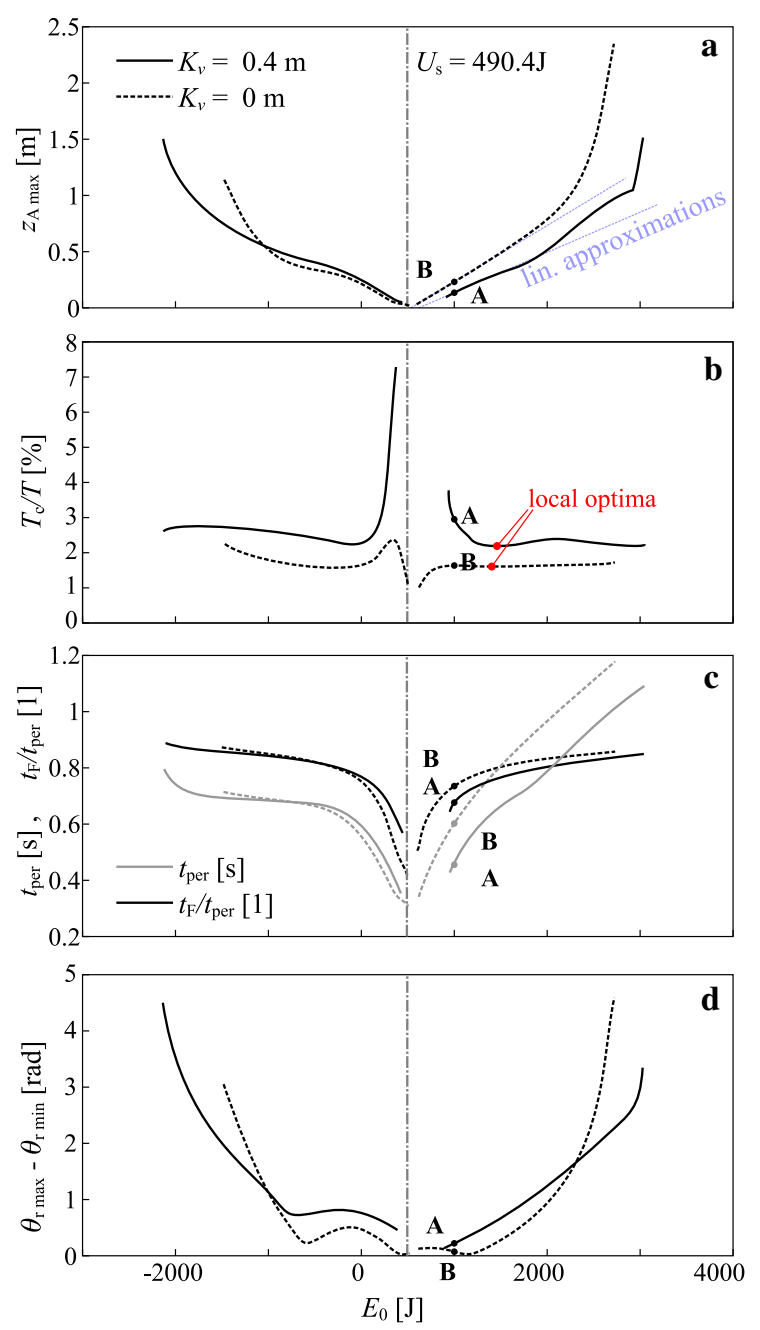

Fig. 9 The dependence of some key indicators on the target energy level $E_{0}$. a Apex height of the trajectory with different parameters; $\mathbf{b}$ ratio of CMSKE to the total kinetic energy $T$; $\mathbf{c}$ time period and flight phase time to time period ratio; and $\mathbf{d}$ angle range $\theta_{\mathrm{r} \max }-\theta_{\mathrm{r} \min }$ of the rotation wheel

as a linear function of $E_{0}$ (light blue lines). Moreover, the hopping height for cases $\mathbf{A}$ and $\mathbf{B}$, i.e., the elevation of the CoM, is realistic according to $[38,39]$, showing that the generated motion is indeed a good replication of human hopping.

The ratio of CMSKE to the total kinetic energy $T$ is plotted in Fig. $9 \mathrm{~b}$ as the function of $E_{0}$. It is possible to find the optimal parameter set where the ratio of CMSKE is the smallest. The ratio $T_{\mathrm{c}} / T$ can therefore be used as a cost function during the controller design of artificial running and walking systems. We can also deduce that the model can be applied for studying the energetic costs of human locomotion.

Figure $9 \mathrm{c}$ shows the time period $t_{\mathrm{per}}$ and the ratio $t_{\mathrm{F}} / t_{\text {per }}$ of the flight phase time duration and the full period. This ratio $t_{\mathrm{F}} / t_{\mathrm{per}}$ is about $70 \%$ and $75 \%$ for cases $\mathbf{A}$ and $\mathbf{B}$, which are close to typical values for human running [24]. Finally, panel d of Fig. 9 shows the range of angular oscillation of the reaction wheel, which plays the role of the torso of the runner. For both cases $\mathbf{A}$ and $\mathbf{B}$, the angle $\theta_{\mathrm{r} \text { max }}-\theta_{\mathrm{r} \text { min }}$ is smaller than $12^{\circ}$, which again agrees with realistic human running.

\subsection{Biomechanical effect of foot positioning}

To demonstrate that model and analysis methods presented in this paper can be used to analyze the effect of biomechanical parameters on the locomotion performance, we focus on the effect of pre-impact tiptoe positioning on locomotion velocity and impact intensity. To this end, the range of investigated control parameters is extended to higher energy levels in order to have a more global view of the applicable parameter set.

The dependence of locomotion velocity $\bar{v}$ on the tiptoe positioning, characterized by the distance $x_{\mathrm{A}}-x_{\mathrm{G}}$, is depicted in panel a of Fig. 10 for five different levels of the energy $E_{0}$. It can be seen that the relation between $\bar{v}$ and the tiptoe positioning is almost linear for each value of $E_{0}$. This observation indicates that footlanding in front of the $\mathrm{CoM}$ is beneficial during running, because it results in a higher locomotion velocity. This case, when $x_{\mathrm{A}}-x_{\mathrm{G}}>0$, is referred to as overstriding in the literature [20]. Figure 10b shows the effect of tiptoe positioning on the CMSKE, which is an indicator of ground-foot impact intensity and impacting forces $[5,26]$. For negative values of $x_{\mathrm{A}}-x_{\mathrm{G}}$ the kinetic energy ratio $T_{\mathrm{c}} / T$ is small (around $1 \%$ ), while it tends to be higher (up to $4 \%$ ) for positive values (i.e., when overstriding). The general consequence for athletes is that foot-landing in front of the CoM induces high kinetic energy loss and, therefore, high intensity ground-foot collisions and low energy efficiency. In other words, overstriding is disadvantageous in this respect, which is the opposite outcome from the velocity consideration represented in Fig. 10a. Hence, a runner has to find the balance between energy efficiency and running speed: short-distance sprinters may touch down their tiptoe in front of the CoM in order to maximize speed, while 


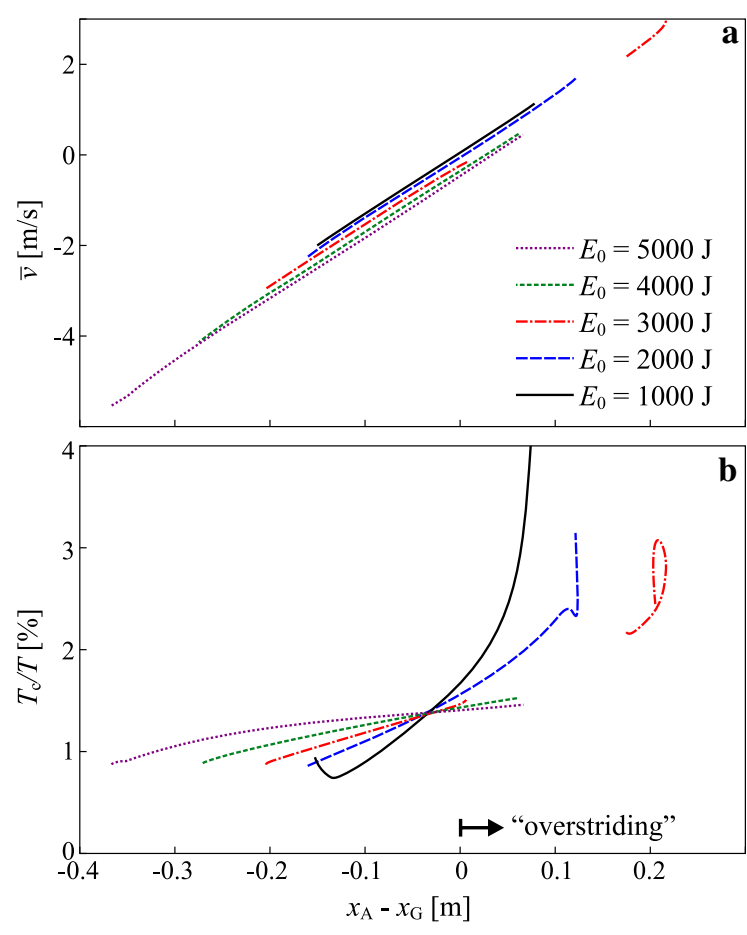

Fig. 10 Average locomotion velocity $\bar{v}$ and CMSKE (as given by $T_{\mathrm{c}}$ ) as functions of the horizontal tiptoe position $x_{\mathrm{A}}-x_{\mathrm{G}}$ relative to the body $\mathrm{CoM}$

long distance runners may avoid overstriding in order to reduce energetic costs.

\section{Conclusions and further steps}

A planar three-segmented leg model was analyzed, which takes into account the inelastic ground-foot impact-induced energy absorption and an active controller, which maintains a uniform energy level. We showed that there exist relative periodic orbits that corresponds to hopping, and that they are stable over a large and realistic parameter range. This was achieved by means of computing the monodromy matrix with a well-established method for piecewise-smooth dynamical systems. We found that both forward and backward motion is possible and that the hopping height can be tuned. Locomotion velocities in the range $(-7,3) \mathrm{m} / \mathrm{s}$ and tiptoe elevations in range $(0.01,4) \mathrm{m}$ were discovered by tuning the main control parameters $E_{0}$ and $K_{v}$ only, while the remaining parameters we have chosen to guarantee stable operation in a wide region of $E_{0}$ and $K_{v}$. We further showed that it is possible to tune the parameters to achieve optimal motion, not only in the sense of lowest foot impact intensity (i.e., energy loss), but also in the sense of apex height, stability and robustness. Overall, this demonstrates the usefulness of the model and our analysis approach for obtaining insights into the biomechanics of running.

Future work will concentrate on tuning control parameters to achieve better performance and a larger region of stability of forward hopping. In this way, we intend to demonstrate that the presented methodology and results can be applied also to more complex models of legged locomotion. The longer term goal is to develop the model further toward more humanlike motion; in particular, two legs and a more accurate model for the upper body can be considered. Furthermore, ground contact of multiple points of the foot will be included in the model. Such a further developed model can also be used to investigate the effect of the terrain on the optimal motion, for instance, by considering inclination of the ground.

Although some conclusion regarding human motion can be drawn from mathematically generated trajectories of a model, a comparison with laboratory experiments with human subjects remains essential. After refinement of the proposed mechanical model and the controller, the determination of other performance measures of human running may become feasibleenabling fine-tuning against measured data.

Acknowledgements Open access funding provided by Budapest University of Technology and Economics (BME). The research reported in this paper was supported by the Higher Education Excellence Program of the Ministry of Human Capacities in the frame of Biotechnology research area of Budapest University of Technology and Economics (BME FIKP-BIO) and by the Hungarian National Research, Development and Innovation Office (Project id.: NKFI-FK18 128636).

\section{Compliance with ethical standards}

Conflict of interest The authors declare that they have no conflict of interest.

Funding This study was funded by MTA-BME Lendület Human Balancing Research Group, Hungary.

Open Access This article is distributed under the terms of the Creative Commons Attribution 4.0 International License (http:// creativecommons.org/licenses/by/4.0/), which permits unrestricted use, distribution, and reproduction in any medium, provided you give appropriate credit to the original author(s) and the source, provide a link to the Creative Commons license, and indicate if changes were made. 


\section{References}

1. Dziewiecki, K., Blajer, W., Mazur, Z., Czaplicki, A.: Modeling and computational issues in the inverse dynamics simulation of triple jump. Multibody Syst. Dyn. 32(3), 299316 (2014). https://doi.org/10.1007/s11044-013-9375-6

2. Seyfarth, A., Günther, M., Blickhan, R.: Stable operation of an elastic three-segment leg. Biol. Cybern. 84(5), 365-382 (2001)

3. Piiroinen, P.T., Dankowicz, H.J.: Low-cost control of repetitive gait in passive bipedal walkers. Int. J. Bifurc. Chaos 15(6), 1959-1973 (2005). https://doi.org/10.1142/ S0218127405013083

4. Westervelt, E.R., Grizzle, J.W., Chevallereau, C., et al.: Feedback Control of Dynamic Bipedal Robot Locomotion. CRC Press, Boca Raton (2007)

5. Kövecses, J., Kovács, L.L.: Foot impact in different modes of running: mechanisms and energy transfer. In: Procedia IUTAM 2 (Symposium on Human Body Dynamics, 2011), pp. 101-108 (2011)

6. Zelei, A., Bencsik, L., Kovács, L.L., Stépán, G.: Energy efficient walking and running-impact dynamics based on varying geometric constraints. In: 12th Conference on Dynamical Systems Theory and Applications, Lodz, Poland, pp. 259-270 (2013)

7. Johnson, A.M., Koditschek, D.E.: Legged selfmanipulation. IEEE Access 1, 310-334 (2013). https://doi. org/10.1109/ACCESS.2013.2263192

8. Johnson, A.M., Burden, S.A., Koditschek, D.E.: A hybrid systems model for simple manipulation and selfmanipulation systems. Int. J. Robot. Res. 35(11), 1354-1392 (2016). https://doi.org/10.1177/0278364916639380

9. Bencsik, L., Zelei, A.: Running form analysis based on impact dynamics: a minimally complex mechanical model. Periodica Polytech. Mech. Eng. 63(1), 7-15 (2019). https:// doi.org/10.3311/PPme.11746

10. Beer, R.D.: Beyond control: the dynamics of brain-bodyenvironment interaction in motor systems. Adv. Exp. Med. Biol. 629, 7-24 (2009). https://doi.org/10.1007/ 978-0-387-77064-2_2

11. Stépán, G.: Delay effects in the human sensory system during balancing. Trans. R. Soc. A 367(1981), 1195-1212 (2009)

12. Czaplicki, A., Silva, M.T., Ambrósio, J.C.: Biomechanical modelling for whole body motion using natural coordinates. J. Theor. Appl. Mech. 42(4), 927-944 (2004)

13. Rajagopal, A., Dembia, C., DeMers, M., Delp, D., Hicks, J., Delp, S.: Full-body musculoskeletal model for muscledriven simulation of human gait. IEEE Trans. Biomed. Eng. https://doi.org/10.1109/TBME.2016.2586891

14. Insperger, T., Milton, J., Stépán, G.: Acceleration feedback improves balancing against reflex delay. J. R. Soc. Interface 10(79), Article No. 20120763 (2013)

15. Holmes, P., Full, R.J., Koditschek, D.E., Guckenheimer, J.: The dynamics of legged locomotion: models, analyses, and challenges. SIAM Rev. 48(2), 207-304 (2006)

16. Rummel, J., Seyfarth, A.: Stable running with segmented legs. Int. J. Robot. Res. 27(8), 919-934 (2008)

17. Zelei, A., Krauskopf, B., Insperger, T.: Control optimization for a three-segmented hopping leg model of human loco- motion. In: DSTA 2017-Vibration, Control and Stability of Dynamical Systems, Lodz, Poland 599-610 (11-14, December 2017)

18. Jungers, W.L.: Barefoot running strikes back. Nat. Biomech. 463(7280), 433-434 (2010)

19. Lieberman, D.E., Venkadesan, M., Werbel, W.A., Daoud, A.I., D'Andrea, S., Davis, I.S., Mang'Eni, R.O., Pitsiladis, Y.: Foot strike patterns and collision forces in habitually barefoot versus shod runners. Nat. Biomech. 463(7280), 531-535 (2010). https://doi.org/10.1038/nature08723

20. Souza, R.B.: An evidence-based videotaped running biomechanics analysis. Phys. Med. Rehabil. Clinics N. Am. 27(1), 217-236 (2016). https://doi.org/10.1016/j.pmr.2015.08.006

21. Chagdes, J., Rietdyk, S., Jeffrey, M., Howard, N., Raman, A.: Dynamic stability of a human standing on a balance board. J. Biomech. 46(15), 2593-2602 (2013)

22. Insperger, T., Milton, J.: Sensory uncertainty and stick balancing at the fingertip. Biol. Cybern. 108(1), 85-101 (2016). https://doi.org/10.1007/s00422-013-0582-2

23. Fekete, L., Krauskopf, B., Zelei, A.: Three-segmented hopping leg for the analysis of human running locomotion. In: The 9th European Nonlinear Dynamics Conference (ENOC 2017), Budapest, Hungary, pp. 1-2 (2017)

24. Novacheck, T.F.: The biomechanics of running. Gait Posture 7(1), 77-95 (1998). https://doi.org/10.1016/ S0966-6362(97)00038-6

25. Garcia, M., Chatterjee, A., Ruina, A., Coleman, M.: The simplest walking model: stability, complexity, and scaling. J. Biomech. Eng. 120, 281-288 (1998)

26. Kövecses, J., Font-Llagunes, J.M.: An eigenvalue problem for the analysis of variable topology mechanical systems. ASME J. Comput. Nonlinear Dyn. 4(3), 9 (2009). https:// doi.org/10.1115/1.3124784

27. Van der Schaft, A.J., Schumacher, H.: An Introduction to Hybrid Dynamical Systems. Springer, Berlin (2000); ISBN 978-1-85233-233-4

28. Bernardo, M., Budd, C., Champneys, A.R., Kowalczyk, P.: Piecewise-smooth Dynamical Systems - Theory and Applications. Springer, Berlin (2008); ISBN 978-1-84628-039-9

29. Yamakita, M., Asano, F.: Extended passive velocity field control with variable velocity fields for a kneed biped. Adv. Robot. 15(2), 139-168 (2001). https://doi.org/10.1163/ 15685530152116209

30. Dankowicz, H.J., Piiroinen, P.T.: Exploiting discontinuities for stabilization of recurrent motions. Dyn. Syst. 17(4), 317-342 (2002). https://doi.org/10.1080/ 1468936021000041663

31. de Jalón, J., Bayo, E.: Kinematic and Dynamic Simulation of Multibody Systems: The Real-Time Challenge. Springer, Berlin (1994)

32. Kövecses, J., Piedoboeuf, J.C., Lange, C.: Dynamic modeling and simulation of constrained robotic systems. IEEE/ASME Trans. Mechatron. 2(2), 165-177 (2003)

33. Chi, K.J., Schmitt, D.: Mechanical energy and effective foot mass during impact loading of walking and running. J. Biomech. 38(7), 1387-1395 (2005). https://doi.org/10. 1016/j.jbiomech.2004.06.020

34. Leine, R.I., van Campen, D.H.: Discontinuous bifurcations of periodic solutions. Math. Comput. Model. 36(3), 259-273 (2002). https://doi.org/10.1016/S0895-7177(02)00124-3 
35. Müller, P.C.: Calculation of lyapunov exponents for dynamic systems with discontinuities. Chaos Solitons Fract. 5(9), 1671-1681 (1995). https://doi.org/10.1016/ 0960-0779(94)00170-U

36. Awrejcewicz, J., Kudra, G.: Stability analysis and lyapunov exponents of a multi-body mechanical system with rigid unilateral constraints. Nonlinear Anal. Theory Methods Appl. 63(5-7), e909-e918 (2005). https://doi.org/10.1016/ j.na.2004.12.038

37. de Leva, P.: Adjustments to Zatsiorsky-Seluyanov's segment inertia parameters. J. Biomech. 29(9), 1223-1230 (1996)

38. Vanezis, A., Lees, A.: A biomechanical analysis of good and poor performers of the vertical jump. In: Ergonomics, Issue 11-14: Sports, Leisure and Ergonomics (SLE) Conference, 19-21 November 2003, vol. 48(11-14), pp. 1594-1603 (2005). https://doi.org/10.1080/00140130500101262
39. Kamandulis, S., Venckunas, T., Snieckus, A., Nickus, E., Stanislovaitiene, J., Skurvydas, A.: Changes of vertical jump height in response to acute and repetitive fatiguing conditions. Sci. Sports 31(6), 163-171 (2016). https://doi. org/10.1016/j.scispo.2015.11.004

Publisher's Note Springer Nature remains neutral with regard to jurisdictional claims in published maps and institutional affiliations. 